\title{
On a fractional problem of Lane-Emden type: Ulam type stabilities and numerical behaviors
}

\author{
Kamel Tablennehas ${ }^{1}$, Zoubir Dahmani ${ }^{1}$, Meriem Mansouria Belhamiti', Amira Abdelnebi ${ }^{1}$ and
} Mehmet Zeki Sarikaya ${ }^{2^{*}}$ (i)

${ }^{*}$ Correspondence:

sarikayamz@gmail.com

${ }^{2}$ Department of Mathematics,

Faculty of Science and Arts, Düzce

University, Düzce, Turkey

Full list of author information is

available at the end of the article

\begin{abstract}
In this work, we study some types of Ulam stability for a nonlinear fractional differential equation of Lane-Emden type with anti periodic conditions. Then, by using a numerical approach for the Caputo derivative, we investigate behaviors of the considered problem.
\end{abstract}

MSC: $30 C 45 ; 39 B 72 ; 39 B 82$

Keywords: Lane-Emden equation; Caputo derivative; Random differential equation; Ulam stability; Fourth Runge-Kutta method

\section{Introduction}

The theory of singular fractional boundary value problems has become an area of research investigation in the last three decades (see $[1,3,6,7,16,21])$. One of the equations describing this type of problems is the very important Lane-Emden equation, which was published by Lane in 1870 [18] and detailed by Emden [8]. Lane-Emden differential equations are singular initial value problems of the second order, they describe a variety of phenomena in mathematical physics and astrophysics such as aspects of the stellar structure. For more information and some applications, one can consult Refs. [2, 13, 23].

The classical Lane-Emden equation has the following form [5, 8]:

$$
x^{\prime \prime}(t)+\frac{a}{t} x^{\prime}(t)+f(t, x(t))=g(t), \quad t \in[0,1],
$$

under the conditions

$$
x(0)=A, \quad x^{\prime}(0)=B,
$$

where $A$ and $B$ are constants and $f$ and $g$ are continuous real functions.

(c) The Author(s) 2021. This article is licensed under a Creative Commons Attribution 4.0 International License, which permits use, sharing, adaptation, distribution and reproduction in any medium or format, as long as you give appropriate credit to the original author(s) and the source, provide a link to the Creative Commons licence, and indicate if changes were made. The images or other third party material in this article are included in the article's Creative Commons licence, unless indicated otherwise in a credit line to the material. If material is not included in the article's Creative Commons licence and your intended use is not permitted by statutory regulation or exceeds the permitted use, you will need to obtain permission directly from the copyright holder. To view a copy of this licence, visit http://creativecommons.org/licenses/by/4.0/ 
The above problem has attracted many researchers attention. In fact, in [20], the authors have used the method of collocation to study the following Lane-Emden problem:

$$
\left\{\begin{array}{l}
D^{\alpha} y(t)+\frac{k}{t^{\alpha-\beta}} D^{\beta} y(t)+f(t, y(t))=g(t), \quad t \in[0,1] \\
k \geq 0, \quad 1<\alpha \leq 2, \quad 0<\beta \leq 1
\end{array}\right.
$$

Ibrahim [15] has been concerned with the stability of Ulam Hyers for the following fractional Lane-Emden problem:

$$
\left\{\begin{array}{l}
D^{\beta}\left(D^{\alpha}+\frac{a}{t}\right) u(t)+f(t, u(t))=g(t) \\
u(0)=\mu, \quad u(1)=v, \\
0<\alpha, \beta \leq 1, \quad 0 \leq t \leq 1, \quad a \geq 0
\end{array}\right.
$$

under the conditions: $D^{\gamma}$ is the Caputo derivative, $f$ is a continuous function and $g \in$ $C([0,1])$.

Very recently, Y. Gouari et al. [10] have investigated the following nonlocal fractional problem of Lane-Emden type:

$$
\left\{\begin{array}{l}
D^{\beta}\left(D^{\alpha}+\frac{k}{t^{\lambda}}\right) y(t)+\Delta_{1} f\left(t, y(t), D^{\delta} y(t)\right)+\Delta_{2} g\left(t, y(t), I^{\rho} y(t)\right)+h(t, y(t))=l(t), \\
y(0)=0, \quad y(1)=b \int_{0}^{\eta} y(s) d s, \quad 0<\eta<1, \quad I^{q} y(u)=y(1), \quad 0<u<1, \\
k>0, \quad 0<\lambda \leq 1, \quad 1 \leq \beta \leq 2, \quad 0 \leq \alpha, \delta \leq 1, \quad t \in] 0,1[
\end{array}\right.
$$

Motivated by the above cited papers, in [25] we have proved the existence and uniqueness of solutions by application of the Banach contraction principle for the following anti periodic fractional differential problem:

$$
\left\{\begin{array}{l}
D^{\alpha} D^{\beta} y(t)+\frac{k}{t^{\lambda}} D^{\alpha} y(t)+a_{1} F\left(t, y(t), D^{\gamma} y(t), J^{p} y(t)\right) \\
\quad+a_{2} G\left(t, y(t), D^{\gamma} y(t)\right)+a_{3} H(t, y(t))=L(t) . \\
y(0)+y(1)=0, \quad y^{\prime}(0)+y^{\prime}(1)=0, \quad D^{\gamma}(0)+D^{\gamma}(1)=0, \\
k>0, \quad 1 \leq \beta \leq 2, \quad 0 \leq \gamma \leq \alpha \leq 1, \quad 0<\lambda<1, \quad p>0, \quad t \in[0,1],
\end{array}\right.
$$

where $I:=[0,1]$, the derivatives of the problem are in the sense of Caputo, $J^{p}$ denotes the Riemann-Liouville integral of order $p$ and $F: I \times \mathbb{R}^{3} \rightarrow \mathbb{R}, G: I \times \mathbb{R}^{2} \rightarrow \mathbb{R}, H: I \times \mathbb{R}^{2} \rightarrow \mathbb{R}$ and $L: I \rightarrow \mathbb{R}$ are four given functions, and $\mathbb{R}$ being the set of real numbers.

In this work, we continue studying the above problem by investigating certain types of Ulam stability for the problem (1). Then, using a numerical approach of the derivative Caputo, we analyze certain behavior of the problem by means of the fourth-order RungeKutta integrator method.

\section{Preliminaries}

We present some necessary lemmas and theorems which will be used in this paper.

As it is proved in our last work [25], the integral solution of (1) is given by the following auxiliary result. 
Lemma 1 Let $L_{1} \in C([0,1]), t \in I, 0 \leq \gamma \leq \alpha \leq 1,1<\beta<2$. Then the integral solution of the problem

$$
\left\{\begin{array}{l}
D^{\alpha}\left(D^{\beta}\right) y(t)+\left(\frac{k}{t^{\lambda}}\right) D^{\alpha} y(t)=L_{1}(t), \\
y(0)+y(1)=0, \quad y^{\prime}(0)+y^{\prime}(1)=0, \quad D^{\gamma}(0)+D^{\gamma}(1)=0
\end{array}\right.
$$

is given by the following expression:

$$
\begin{aligned}
y(t)= & \int_{0}^{t} \frac{(t-s)^{\beta-1}}{\Gamma(\beta)} \int_{0}^{s} \frac{(s-u)^{\alpha-1}}{\Gamma(\alpha)}\left[L_{1}(u)-\frac{k}{u^{\lambda}} D^{\alpha} y(u)\right] d u d s \\
& +\left[K_{1} t^{\beta}+K_{2} t+K_{3}\right]\left[\int_{0}^{1} \frac{(1-s)^{\beta-2}}{\Gamma(\beta-1)} \int_{0}^{s} \frac{(s-u)^{\alpha-1}}{\Gamma(\alpha)}\left[L_{1}(u)-\frac{k}{u^{\lambda}} D^{\alpha} y(u)\right] d u d s\right] \\
& +\left[K_{4} t^{\beta}+K_{5} t-K_{6}\right]\left[\int_{0}^{1} \frac{(1-s)^{\beta-\gamma-1}}{\Gamma(\beta-\gamma)} \int_{0}^{s} \frac{(s-u)^{\alpha-1}}{\Gamma(\alpha)}\left[L_{1}(u)-\frac{k}{u^{\lambda}} D^{\alpha} y(u)\right] d u d s\right] \\
& -\left[K_{7}\right]\left[\int_{0}^{1} \frac{(1-s)^{\beta-1}}{\Gamma(\beta)} \int_{0}^{s} \frac{(s-u)^{\alpha-1}}{\Gamma(\alpha)}\left[L_{1}(u)-\frac{k}{u^{\lambda}} D^{\alpha} y(u)\right] d u d s\right],
\end{aligned}
$$

where

$$
\begin{aligned}
L_{1}(u)= & L(u)-a_{1} F\left(u, y(u), D^{\gamma} y(u), J^{p} y(u)\right)-a_{2} G\left(u, y(u), D^{\gamma} y(u)\right) \\
& -a_{3} H(u, y(u))-\frac{k}{u^{\lambda}} D^{\alpha} y(u)
\end{aligned}
$$

and

$$
\begin{aligned}
K_{1} & =\frac{\Gamma(\beta-\gamma+1)}{\beta[\Gamma(\beta-\gamma+1)-2 \Gamma(\beta) \Gamma(2-\gamma)]}, \\
K_{2} & =\frac{\Gamma(\beta) \Gamma(2-\gamma)}{2 \Gamma(\beta) \Gamma(2-\gamma)-\Gamma(\beta-\gamma+1)}, \\
K_{3} & =\frac{\Gamma(\beta+1) \Gamma(2-\gamma)-\Gamma(\beta-\gamma+1)}{2 \beta \Gamma(\beta-\gamma+1)-4 \Gamma(\beta+1) \Gamma(2-\gamma)}, \\
K_{4} & =\frac{2 \Gamma(2-\gamma) \Gamma(\beta-\gamma+1)}{\beta[\Gamma(\beta-\gamma+1)-2 \Gamma(\beta) \Gamma(2-\gamma)]}, \\
K_{5} & =\frac{\Gamma(2-\gamma) \Gamma(\beta-\gamma+1)}{2 \Gamma(\beta) \Gamma(2-\gamma)-\Gamma(\beta-\gamma+1)}, \\
K_{6} & =\frac{2 \Gamma(2-\gamma) \Gamma(\beta-\gamma+1)-\beta \Gamma(2-\gamma) \Gamma(\beta-\gamma+1)}{2 \beta \Gamma(\beta-\gamma+1)-4 \Gamma(\beta+1) \Gamma(2-\gamma)}, \\
K_{7} & =\frac{\Gamma(\beta-\gamma+1)-2 \Gamma(\beta) \Gamma(2-\gamma)}{2 \Gamma(\beta-\gamma+1)-4 \Gamma(\beta) \Gamma(2-\gamma)} .
\end{aligned}
$$

Before presenting our main results, we shall introduce also the Banach space

$$
X:=\left\{y \in C(I, \mathbb{R}), D^{\alpha} y \in C(I, \mathbb{R}), D^{\gamma} y \in C(I, \mathbb{R})\right\},
$$

and the norm

$$
\|y\|_{X}=\max \left\{\|y\|_{\infty},\left\|D^{\alpha} y\right\|_{\infty},\left\|D^{\gamma} y\right\|_{\infty}\right\},
$$


where

$$
\|x\|_{\infty}=\sup _{t \in I}|x(t)|, \quad\left\|D^{\alpha} x\right\|_{\infty}=\sup _{t \in I}\left|D^{\alpha} x(t)\right|, \quad\left\|D^{\gamma} x\right\|_{\infty}=\sup _{t \in I}\left|D^{\gamma} x(t)\right| .
$$

Also, we consider the following hypotheses:

(H1): There exist nonnegative constants $W_{i}, i=1, \ldots, 6$, such that for each $t \in I$ and for all $x_{1}, x_{2}, x_{3}, y_{1}, y_{2}, y_{3} \in \mathbb{R}$ we have

$$
\begin{aligned}
& \left|F\left(t, x_{1}, x_{2}, x_{3}\right)-F\left(t, y_{1}, y_{2}, y_{3}\right)\right| \leq W_{1}\left|x_{1}-y_{1}\right|+W_{2}\left|x_{2}-y_{2}\right|+W_{3}\left|x_{3}-y_{3}\right|, \\
& \left|G\left(t, x_{1}, x_{2}\right)-G\left(t, y_{1}, y_{2}\right)\right| \leq W_{4}\left|x_{1}-y_{1}\right|+W_{5}\left|x_{2}-y_{2}\right|, \\
& \left|H\left(t, x_{1}\right)-H\left(t, y_{1}\right)\right| \leq W_{6}\left|x_{1}-y_{1}\right| .
\end{aligned}
$$

The following quantities are also needed in this paper:

$$
\begin{aligned}
N_{1}= & \left(a_{1} W_{1,2}+a_{2} W_{4,5}+a_{3} W_{6}\right)\left[\frac{1+\left|K_{7}\right|}{\Gamma(\alpha+\beta+1)}\right. \\
& \left.+\frac{\left|K_{1}\right|+\left|K_{2}\right|+\left|K_{3}\right|}{\Gamma(\alpha+\beta)}+\frac{\left|K_{4}\right|+\left|K_{5}\right|+\left|K_{6}\right|}{\Gamma(\alpha+\beta-\gamma+1)}\right] \\
& +a_{1} W_{3}\left[\frac{1+\left|K_{7}\right|}{\Gamma(\alpha+\beta+p+1)}+\frac{\left|K_{1}\right|+\left|K_{2}\right|+\left|K_{3}\right|}{\Gamma(\alpha+\beta+p)}+\frac{\left|K_{4}\right|+\left|K_{5}\right|+\left|K_{6}\right|}{\Gamma(\alpha+\beta-\gamma+p+1)}\right] \\
& +|k| \Gamma(1-\lambda)\left[\frac{1+\left|K_{7}\right|}{\Gamma(\alpha+\beta-\lambda+1)}+\frac{\left|K_{1}\right|+\left|K_{2}\right|+\left|K_{3}\right|}{\Gamma(\alpha+\beta-\lambda)}+\frac{\left|K_{4}\right|+\left|K_{5}\right|+\left|K_{6}\right|}{\Gamma(\alpha+\beta-\gamma-\lambda+1)}\right], \\
N_{2}= & \left(a_{1} W_{1,2}+a_{2} W_{4,5}+a_{3} W_{6}\right)\left[\frac{1+\left|K_{7}\right|}{\Gamma(\beta+1)}+\frac{\left|K_{1}\right| \Gamma(\beta+1) \Gamma(2-\alpha)+\left|K_{2}\right| \Gamma(\beta-\alpha+1)}{\Gamma(\beta) \Gamma(\beta-\alpha+1) \Gamma(2-\alpha)}\right. \\
& \left.+\frac{\left|K_{4}\right| \Gamma(\beta+1) \Gamma(2-\alpha)+\left|K_{5}\right| \Gamma(\beta-\alpha+1)}{\Gamma(\beta-\gamma+1) \Gamma(\beta-\alpha+1) \Gamma(2-\alpha)}\right] \\
& +a_{1} W_{3}\left[\frac{1+\left|K_{7}\right|}{\Gamma(\beta+p+1)}+\frac{\left|K_{1}\right| \Gamma(\beta+1) \Gamma(2-\alpha)+\left|K_{2}\right| \Gamma(\beta-\alpha+1)}{\Gamma(\beta+p) \Gamma(\beta-\alpha+1) \Gamma(2-\alpha)}\right. \\
& \left.+\frac{\left|K_{4}\right| \Gamma(\beta+1) \Gamma(2-\alpha)+\left|K_{5}\right| \Gamma(\beta-\alpha+1)}{\Gamma(\beta-\gamma+p+1) \Gamma(\beta-\alpha+1) \Gamma(2-\alpha)}\right] \\
& +|k| \Gamma(1-\lambda)\left[\frac{1+\left|K_{7}\right|}{\Gamma(\beta-\lambda+1)}+\frac{\left|K_{1}\right| \Gamma(\beta+1) \Gamma(2-\alpha)+\left|K_{2}\right| \Gamma(\beta-\alpha+1)}{\Gamma(\beta-\lambda) \Gamma(\beta-\alpha+1) \Gamma(2-\alpha)}\right. \\
& \left.+\frac{\left|K_{4}\right| \Gamma(\beta+1) \Gamma(2-\alpha)+\left|K_{5}\right| \Gamma(\beta-\alpha+1)}{\Gamma(\beta-\gamma-\lambda+1) \Gamma(\beta-\alpha+1) \Gamma(2-\alpha)}\right] \\
& \left.+\frac{\left|K_{4}\right| \Gamma(\beta+1) \Gamma(2-\gamma)+\left|K_{5}\right| \Gamma(\beta-\gamma+1)}{\Gamma(\alpha+\beta-2 \gamma+1) \Gamma(\beta-\gamma+1) \Gamma(2-\gamma)}\right] \\
N_{3}= & \left(a_{1} W_{1,2}+a_{2} W_{4,5}+a_{3} W_{6}\right)\left[\frac{1+\left|K_{7}\right|}{\Gamma(\alpha+\beta-\gamma+1)}\right. \\
& \frac{\left|K_{1}\right| \Gamma(\beta+1) \Gamma(2-\gamma)+\left|K_{2}\right| \Gamma(\beta-\gamma+1)}{\Gamma(\alpha+\beta-\gamma) \Gamma(\beta-\gamma+1) \Gamma(2-\gamma)} \\
&
\end{aligned}
$$




$$
\begin{aligned}
& \left.+\frac{\left|K_{4}\right| \Gamma(\beta+1) \Gamma(2-\gamma)+\left|K_{5}\right| \Gamma(\beta-\gamma+1)}{\Gamma(\alpha+\beta-2 \gamma+p+1) \Gamma(\beta-\gamma+1) \Gamma(2-\gamma)}\right] \\
& +|k| \Gamma(1-\lambda)\left[\frac{1+\left|K_{7}\right|}{\Gamma(\alpha+\beta-\gamma-\lambda+1)}+\frac{\left|K_{1}\right| \Gamma(\beta+1) \Gamma(2-\gamma)+\left|K_{2}\right| \Gamma(\beta-\gamma+1)}{\Gamma(\alpha+\beta-\gamma-\lambda) \Gamma(\beta-\gamma+1) \Gamma(2-\gamma)}\right. \\
& \left.+\frac{\left|K_{4}\right| \Gamma(\beta+1) \Gamma(2-\gamma)+\left|K_{5}\right| \Gamma(\beta-\gamma+1)}{\Gamma(\alpha+\beta-2 \gamma-\lambda+1) \Gamma(\beta-\gamma+1) \Gamma(2-\gamma)}\right],
\end{aligned}
$$

where $W_{1,2}:=\max \left(W_{1}, W_{2}\right)$ and $W_{4,5}:=\max \left(W_{4}, W_{5}\right)$.

We recall the following result [25], which allows us to study the stability phenomena of the considered problem.

Theorem 2 ([25]) Assume that (H1) holds and suppose that $0<N<1$, where $N=$ $\max \left(N_{1}, N_{2}, N_{3}\right)$. Then the problem (1) has a unique solution on $I$.

\section{Ulam type stabilities}

The notion of the stability problem of functional equations originated from a problem of Stanislaw Ulam [26], posed in 1940: When can we assert that approximate solution of a functional equation can be approximated by a solution of the corresponding equation. In 1941, Hyers [14] solved it. This approach can guarantee that there exists a close exact solution useful in many applications. For more details on the recent advances on the Hyers-Ulam stability (see for example [9, 11, 24, 27]).

In order to study some types of Ulam stability for the problem (1), we consider the following fractional differential equation:

Let $1 \leq \beta \leq 2,0 \leq \gamma \leq \alpha \leq 1$ and $\epsilon$ a positive real numbers and the function $T \in$ $C\left(I, \mathbb{R}^{+}\right)$. We consider the following fractional differential equation:

$$
\begin{aligned}
& D^{\alpha} D^{\beta} y(t)+\frac{k}{t^{\lambda}} D^{\alpha} y(t)+a_{1} F\left(t, y(t), D^{\gamma} y(t), J^{p} y(t)\right) \\
& \quad+a_{2} G\left(t, y(t), D^{\gamma} y(t)\right)+a_{3} H(t, y(t))=L(t), \quad t \in I,
\end{aligned}
$$

and the following fractional differential inequality:

$$
\begin{aligned}
& \mid D^{\alpha} D^{\beta} x(t)+\frac{k}{t^{\lambda}} D^{\alpha} x(t)+a_{1} F\left(t, x(t), D^{\gamma} x(t), J^{p} x(t)\right)+a_{2} G\left(t, x(t), D^{\gamma} x(t)\right) \\
& +a_{3} H(t, x(t))-L(t) \mid \leq \epsilon, \quad t \in I, \\
& \mid D^{\alpha} D^{\beta} x(t)+\frac{k}{t^{\lambda}} D^{\alpha} x(t)+a_{1} F\left(t, x(t), D^{\gamma} x(t), J^{p} x(t)\right)+a_{2} G\left(t, x(t), D^{\gamma} x(t)\right) \\
& +a_{3} H(t, x(t))-L(t) \mid \leq \epsilon T(t), \quad t \in I, \\
& \mid D^{\alpha} D^{\beta} x(t)+\frac{k}{t^{\lambda}} D^{\alpha} x(t)+a_{1} F\left(t, x(t), D^{\gamma} x(t), J^{p} x(t)\right)+a_{2} G\left(t, x(t), D^{\gamma} x(t)\right) \\
& +a_{3} H(t, x(t))-L(t) \mid \leq T(t), \quad t \in I
\end{aligned}
$$

Definition 3 The problem (1) is Ulam-Hyers stable, if there exists a real number $S>0$, such that, for each $\epsilon>0, t \in I$, and for each $x \in X$ solution of (5), there exists a solution 
$y \in X$ of (4) (with the same conditions as in (1)), such that

$$
\|x-y\|_{X} \leq S \epsilon, \quad t \in I
$$

Definition 4 The problem (1) is generalized Ulam-Hyers stable, if there exists an increasing function $Z \in C\left(\mathbb{R}^{+}, \mathbb{R}^{+}\right), Z(0)=0$, such that, for all $\epsilon>0$, and for each solution $x \in X$ of (5), there exists a solution $y \in X$ of (4) (with the same conditions as in (1)), such that

$$
\|x-y\|_{X} \leq Z(\epsilon), \quad t \in I
$$

Definition 5 The problem (1) is Ulam-Hyers-Rassias stable, if there exists a function $T \in C\left(\mathbb{I}, \mathbb{R}^{+}\right)$and $\sigma>0$, such that for each $\epsilon>0$ and for all solutions $x \in X$ of (6) there exists a solution $y \in X$ of (4) (with the same conditions as in (1)), such that

$$
|x(t)-y(t)| \leq \sigma \epsilon T(t), \quad t \in I .
$$

Definition 6 The problem (1) is generalized Ulam-Hyers-Rassias stable, if there exists a function $T \in C\left(\mathbb{I}, \mathbb{R}^{+}\right)$and $\sigma>0$, such that for all solutions $x \in X$ of (7) there exists a solution $y \in X$ of (4) (with the same conditions as in (1)), such that

$$
|x(t)-y(t)| \leq \sigma T(t), \quad t \in I
$$

Now, we are ready to prove the following result.

Theorem 7 Assume that $(\mathrm{H} 1)$ is fulfilled and $N=\max \left(N_{1}, N_{2}, N_{3}\right)<1$. Then the problem (1) is Ulam-Hyers stable in X.

Proof Let us note

$$
\begin{aligned}
O= & \int_{0}^{t} \frac{(t-s)^{\beta-1}}{\Gamma(\beta)} \int_{0}^{s} \frac{(s-u)^{\alpha-1}}{\Gamma(\alpha)}\left[L(s)-a_{1} F\left(s, x(s), D^{\gamma} x(s), J^{p} x(s)\right)\right. \\
& \left.-a_{2} G\left(s, x(s), D^{\gamma} x(s)\right)-a_{3} H(s, x(s))-\frac{k}{s^{\lambda}} D^{\alpha} x(s)\right] d u d s \\
& +\left[K_{1} t^{\beta}+K_{2} t+K_{3}\right] \int_{0}^{1} \frac{(1-s)^{\beta-2}}{\Gamma(\beta-1)} \int_{0}^{s} \frac{(s-u)^{\alpha-1}}{\Gamma(\alpha)} \\
& \times\left[L(s)-a_{1} F\left(s, x(s), D^{\gamma} x(s), J^{p} x(s)\right)\right. \\
& \left.-a_{2} G\left(s, x(s), D^{\gamma} x(s)\right)-a_{3} H(s, x(s))-\frac{k}{s^{\lambda}} D^{\alpha} x(s)\right] d u d s \\
& +\left[K_{4} t^{\beta}+K_{5} t-K_{6}\right] \int_{0}^{1} \frac{(1-s)^{\beta-\gamma-1}}{\Gamma(\beta-\gamma)} \int_{0}^{s} \frac{(s-u)^{\alpha-1}}{\Gamma(\alpha)} \\
& \times\left[L(s)-a_{1} F\left(s, x(s), D^{\gamma} x(s), J^{p} x(s)\right)\right. \\
& \left.-a_{2} G\left(s, x(s), D^{\gamma} x(s)\right)-a_{3} H(s, x(s))-\frac{k}{s^{\lambda}} D^{\alpha} x(s)\right] d u d s
\end{aligned}
$$




$$
\begin{aligned}
& -\left[K_{7}\right] \int_{0}^{t} \frac{(t-s)^{\beta-1}}{\Gamma(\beta)} \int_{0}^{s} \frac{(s-u)^{\alpha-1}}{\Gamma(\alpha)}\left[L(s)-a_{1} F\left(s, x(s), D^{\gamma} x(s), J^{p} x(s)\right)\right. \\
& \left.-a_{2} G\left(s, x(s), D^{\gamma} x(s)\right)-a_{3} H(s, x(s))-\frac{k}{s^{\lambda}} D^{\alpha} x(s)\right] d u d s \\
& -\int_{0}^{t} \frac{(t-s)^{\beta-1}}{\Gamma(\beta)} \int_{0}^{s} \frac{(s-u)^{\alpha-1}}{\Gamma(\alpha)}\left[L(s)-a_{1} F\left(s, y(s), D^{\gamma} y(s), J^{p} y(s)\right)\right. \\
& \left.-a_{2} G\left(s, y(s), D^{\gamma} y(s)\right)-a_{3} H(s, y(s))-\frac{k}{s^{\lambda}} D^{\alpha} y(s)\right] d u d s \\
& -\left[K_{1} t^{\beta}+K_{2} t+K_{3}\right] \int_{0}^{1} \frac{(1-s)^{\beta-2}}{\Gamma(\beta-1)} \int_{0}^{s} \frac{(s-u)^{\alpha-1}}{\Gamma(\alpha)}\left[L(s)-a_{1} F\left(s, y(s), D^{\gamma} y(s), J^{p} y(s)\right)\right. \\
& \left.-a_{2} G\left(s, y(s), D^{\gamma} y(s)\right)-a_{3} H(s, y(s))-\frac{k}{s^{\lambda}} D^{\alpha} y(s)\right] d u d s \\
& -\left[K_{4} t^{\beta}+K_{5} t-K_{6}\right] \int_{0}^{1} \frac{(1-s)^{\beta-\gamma-1}}{\Gamma(\beta-\gamma)} \int_{0}^{s} \frac{(s-u)^{\alpha-1}}{\Gamma(\alpha)} \\
& \times\left[L(s)-a_{1} F\left(s, y(s), D^{\gamma} y(s), J^{p} y(s)\right)\right. \\
& \left.-a_{2} G\left(s, y(s), D^{\gamma} y(s)\right)-a_{3} H(s, y(s))-\frac{k}{s^{\lambda}} D^{\alpha} y(s)\right] d u d s \\
& +\left[K_{7}\right] \times \int_{0}^{t} \frac{(t-s)^{\beta-1}}{\Gamma(\beta)} \int_{0}^{s} \frac{(s-u)^{\alpha-1}}{\Gamma(\alpha)}\left[L(s)-a_{1} F\left(s, y(s), D^{\gamma} y(s), J^{p} y(s)\right)\right. \\
& \left.-a_{2} G\left(s, y(s), D^{\gamma} y(s)\right)-a_{3} H(s, y(s))-\frac{k}{s^{\lambda}} D^{\alpha} y(s)\right] d u d s \mid \\
& M_{1}=\mid x(t)-\int_{0}^{t} \frac{(t-s)^{\beta-1}}{\Gamma(\beta)} \int_{0}^{s} \frac{(s-u)^{\alpha-1}}{\Gamma(\alpha)}\left[L(s)-a_{1} F\left(s, x(s), D^{\gamma} x(s), J^{p} x(s)\right)\right. \\
& \left.-a_{2} G\left(u, x(s), D^{\gamma} x(s)\right)-a_{3} H(s, x(s))-\frac{k}{s^{\lambda}} D^{\alpha} x(s)\right] d u d s \\
& -\left[K_{1} t^{\beta}+K_{2} t+K_{3}\right] \\
& \times \int_{0}^{1} \frac{(1-s)^{\beta-2}}{\Gamma(\beta-1)} \int_{0}^{s} \frac{(s-u)^{\alpha-1}}{\Gamma(\alpha)}\left[L(s)-a_{1} F\left(s, x(s), D^{\gamma} x(s), J^{p} x(s)\right)\right. \\
& \left.-a_{2} G\left(u, x(s), D^{\gamma} x(s)\right)-a_{3} H(s, x(s))-\frac{k}{s^{\lambda}} D^{\alpha} x(s)\right] d u d s \\
& -\left[K_{4} t^{\beta}+K_{5} t-K_{6}\right] \\
& \times \int_{0}^{1} \frac{(1-s)^{\beta-\gamma-1}}{\Gamma(\beta-\gamma)} \int_{0}^{s} \frac{(s-u)^{\alpha-1}}{\Gamma(\alpha)}\left[L(s)-a_{1} F\left(s, x(s), D^{\gamma} x(s), J^{p} x(s)\right)\right. \\
& \left.-a_{2} G\left(u, x(s), D^{\gamma} x(s)\right)-a_{3} H(s, x(s))-\frac{k}{s^{\lambda}} D^{\alpha} x(s)\right] d u d s \\
& +\left[K_{7}\right] \\
& \times \int_{0}^{t} \frac{(t-s)^{\beta-1}}{\Gamma(\beta)} \int_{0}^{s} \frac{(s-u)^{\alpha-1}}{\Gamma(\alpha)}\left[L(s)-a_{1} F\left(s, x(s), D^{\gamma} x(s), J^{p} x(s)\right)\right. \\
& \left.-a_{2} G\left(u, x(s), D^{\gamma} x(s)\right)-a_{3} H(s, x(s))-\frac{k}{s^{\lambda}} D^{\alpha} x(s)\right] d u d s \mid,
\end{aligned}
$$




$$
\begin{aligned}
& M_{2}=\mid D^{\alpha} x(t)-\int_{0}^{t} \frac{(t-s)^{\beta-1}}{\Gamma(\beta)}\left[L(s)-a_{1} F\left(s, x(s), D^{\gamma} x(s), J^{p} x(s)\right)\right. \\
& \left.-a_{2} G\left(u, x(s), D^{\gamma} x(s)\right)-a_{3} H(s, x(s))-\frac{k}{s^{\lambda}} D^{\alpha} x(s)\right] d s \\
& -\left[\frac{K_{1} \Gamma(\beta+1) t^{\beta-\alpha}}{\Gamma(\beta-\alpha+1)}+\frac{K_{2} t^{1-\alpha}}{\Gamma(2-\alpha)}\right] \\
& \times \int_{0}^{1} \frac{(1-s)^{\beta-2}}{\Gamma(\beta-1)}\left[L(s)-a_{1} F\left(s, x(s), D^{\gamma} x(s), J^{p} x(s)\right)\right. \\
& \left.-a_{2} G\left(s, x(s), D^{\gamma} x(s)\right)-a_{3} H(s, x(s))-\frac{k}{s^{\lambda}} D^{\alpha} x(s)\right] d s \\
& -\left[\frac{K_{4} \Gamma(\beta+1) t^{\beta-\alpha}}{\Gamma(\beta-\alpha+1)}+\frac{K_{5} t^{1-\alpha}}{\Gamma(2-\alpha)}\right] \\
& \times \int_{0}^{1} \frac{(1-s)^{\beta-\gamma-1}}{\Gamma(\beta-\gamma)}\left[L(s)-a_{1} F\left(s, x(s), D^{\gamma} x(s), J^{p} x(s)\right)\right. \\
& \left.-a_{2} G\left(s, x(s), D^{\gamma} x(s)\right)-a_{3} H(s, x(s))-\frac{k}{s^{\lambda}} D^{\alpha} x(s)\right] d s \\
& +\left[K_{7}\right] \int_{0}^{t} \frac{(t-s)^{\beta-1}}{\Gamma(\beta)}\left[L(s)-a_{1} F\left(s, x(s), D^{\gamma} x(s), J^{p} x(s)\right)\right. \\
& \left.-a_{2} G\left(s, x(s), D^{\gamma} x(s)\right)-a_{3} H(s, x(s))-\frac{k}{s^{\lambda}} D^{\alpha} x(s)\right] d s \mid, \\
& M_{3}=\mid D^{\gamma} x(t)-\int_{0}^{t} \frac{(t-s)^{\alpha+\beta-\gamma-1}}{\Gamma(\alpha+\beta-\gamma)}\left[L(s)-a_{1} F\left(s, x(s), D^{\gamma} x(s), J^{p} x(s)\right)-a_{2}\right. \\
& \left.\times G\left(u, x(s), D^{\gamma} x(s)\right)-a_{3} H(s, x(s))-\frac{k}{s^{\lambda}} D^{\alpha} x(s)\right] d s \\
& -\left[\frac{K_{1} \Gamma(\beta+1) t^{\beta-\gamma}}{\Gamma(\beta-\gamma+1)}+\frac{K_{2} t^{1-\gamma}}{\Gamma(2-\gamma)}\right] \\
& \times \int_{0}^{1} \frac{(1-s)^{\alpha+\beta-\gamma-2}}{\Gamma(\alpha+\beta-\gamma-1)}\left[L(s)-a_{1} F\left(s, x(s), D^{\gamma} x(s), J^{p} x(s)\right)\right. \\
& \left.-a_{2} G\left(s, x(s), D^{\gamma} x(s)\right)-a_{3} H(s, x(s))-\frac{k}{s^{\lambda}} D^{\alpha} x(s)\right] d s \\
& -\left[\frac{K_{4} \Gamma(\beta+1) t^{\beta-\gamma}}{\Gamma(\beta-\gamma+1)}+\frac{K_{5} t^{1-\gamma}}{\Gamma(2-\gamma)}\right] \\
& \times \int_{0}^{1} \frac{(1-s)^{\alpha+\beta-2 \gamma-1}}{\Gamma(\alpha+\beta-2 \gamma)}\left[L(s)-a_{1} F\left(s, x(s), D^{\gamma} x(s), J^{p} x(s)\right)\right. \\
& \left.-a_{2} G\left(s, x(s), D^{\gamma} x(s)\right)-a_{3} H(s, x(s))-\frac{k}{s^{\lambda}} D^{\alpha} x(s)\right] d s \\
& +\left[K_{7}\right] \int_{0}^{t} \frac{(t-s)^{\alpha+\beta-\gamma-1}}{\Gamma(\alpha+\beta-\gamma)}\left[L(s)-a_{1} F\left(s, x(s), D^{\gamma} x(s), J^{p} x(s)\right)\right. \\
& \left.-a_{2} G\left(s, x(s), D^{\gamma} x(s)\right)-a_{3} H(s, x(s))-\frac{k}{s^{\lambda}} D^{\alpha} x(s)\right] d s \mid \text {. }
\end{aligned}
$$

Let now $x \in X$ be a solution of (5). Then, by integrating (5), we obtain

$$
M_{1} \leq \frac{\epsilon t^{\alpha+\beta}}{\Gamma(\alpha+\beta+1)}
$$


Thanks to Theorem 2, the unique solution of (1) is given by

$$
\begin{aligned}
y(t)= & \int_{0}^{t} \frac{(t-s)^{\beta-1}}{\Gamma(\beta)} \int_{0}^{s} \frac{(s-u)^{\alpha-1}}{\Gamma(\alpha)}\left[L(s)-a_{1} F\left(s, y(s), D^{\gamma} y(s), J^{p} y(s)\right)\right. \\
& \left.-a_{2} G\left(s, y(s), D^{\gamma} y(s)\right)-a_{3} H(s, y(s))-\frac{k}{s^{\lambda}} D^{\alpha} y(s)\right] d u d s \\
& +\left[K_{1} t^{\beta}+K_{2} t+K_{3}\right] \\
& \times \int_{0}^{1} \frac{(1-s)^{\beta-2}}{\Gamma(\beta-1)} \int_{0}^{s} \frac{(s-u)^{\alpha-1}}{\Gamma(\alpha)}\left[L(s)-a_{1} F\left(s, y(s), D^{\gamma} y(s), J^{p} y(s)\right)\right. \\
& \left.-a_{2} G\left(s, y(s), D^{\gamma} y(s)\right)-a_{3} H(s, y(s))-\frac{k}{s^{\lambda}} D^{\alpha} y(s)\right] d u d s \\
& +\left[K_{4} t^{\beta}+K_{5} t-K_{6}\right] \\
& \times \int_{0}^{1} \frac{(1-s)^{\beta-\gamma-1}}{\Gamma(\beta-\gamma)} \int_{0}^{s} \frac{(s-u)^{\alpha-1}}{\Gamma(\alpha)}\left[L(s)-a_{1} F\left(s, y(s), D^{\gamma} y(s), J^{p} y(s)\right)\right. \\
& \left.-a_{2} G\left(s, y(s), D^{\gamma} y(s)\right)-a_{3} H(s, y(s))-\frac{k}{s^{\lambda}} D^{\alpha} y(s)\right] d u d s \\
& -\left[K_{7}\right] \times \int_{0}^{t} \frac{(t-s)^{\beta-1}}{\Gamma(\beta)} \int_{0}^{s} \frac{(s-u)^{\alpha-1}}{\Gamma(\alpha)}\left[L(s)-a_{1} F\left(s, y(s), D^{\gamma} y(s), J^{p} y(s)\right)\right. \\
& \left.-a_{2} G\left(s, y(s), D^{\gamma} y(s)\right)-a_{3} H(s, y(s))-\frac{k}{s^{\lambda}} D^{\alpha} y(s)\right] d u d s .
\end{aligned}
$$

Then, from all $t \in I$, we get

$$
|x(t)-y(t)| \leq \frac{\epsilon t^{\alpha+\beta}}{\Gamma(\alpha+\beta+1)}+O .
$$

This implies that

$$
\|x-y\|_{\infty} \leq \frac{\epsilon}{\Gamma(\alpha+\beta+1)}+N_{1}\|x-y\|_{X}
$$

By integrating and differentiating (5), we get

$$
M_{2} \leq \frac{\epsilon t^{\beta}}{\Gamma(\beta+1)} .
$$

Similarly, we show that

$$
\left\|D^{\alpha} x-D^{\alpha} y\right\|_{\infty} \leq \frac{\epsilon}{\Gamma(\beta+1)}+N_{2}\|x-y\|_{X}
$$

On the other hand, we have

$$
M_{3} \leq \frac{\epsilon t^{\alpha+\beta-\gamma}}{\Gamma(\alpha+\beta-\gamma+1)} .
$$

Also, we have

$$
\left\|D^{\gamma} x-D^{\gamma} y\right\|_{\infty} \leq \frac{\epsilon}{\Gamma(\alpha+\beta-\gamma+1)}+N_{3}\|x-y\|_{X} .
$$


Using the inequalities (8), (9) and (10), we get

$$
\|x-y\|_{X} \leq \max \left(\frac{\epsilon}{\Gamma(\alpha+\beta+1)}, \frac{\epsilon}{\Gamma(\beta+1)}, \frac{\epsilon}{\Gamma(\alpha+\beta-\gamma+1)}\right)+N\|x-y\|_{X} .
$$

Thus,

$$
\|x-y\|_{X} \leq S \epsilon
$$

such that

$$
S=\frac{\max \left(\frac{1}{\Gamma(\alpha+\beta+1)}, \frac{1}{\Gamma(\beta+1)}, \frac{1}{\Gamma(\alpha+\beta-\gamma+1)}\right)}{1-N}>0 .
$$

Consequently, the problem (1) shows the Ulam-Hyers stability.

Taking $Z(\epsilon)=S \epsilon$, we can state that the problem (1) is generalized Ulam-Hyers stable. In the following, we introduce the following hypothesis to study Rassias stability.

(H2): $T \in C\left(\mathbb{I}, \mathbb{R}^{+}\right)$is continuous, nondecreasing function, and there exists $\lambda_{T, \alpha}>0$ such that $J^{\alpha} T(t) \leq \lambda_{T, \alpha} T(t)$ for each $t \in I$.

We present the following result.

Theorem 8 Assume that $(\mathrm{H} 1)-(\mathrm{H} 2)$ are satisfied and $N:=\max \left(N_{1}, N_{2}, N_{3}\right)<1$.

Then the problem (1) is Ulam-Hyers-Rassias stable in X.

Proof Let $x \in X$ be a solution of (6). Then, by integrating (6), we obtain

$$
M_{1} \leq \epsilon J^{\beta} J^{\alpha} T(t)
$$

Let $y$ be the unique solution of the problem (1). Then, for each $t \in I$, we have

$$
|x(t)-y(t)| \leq \epsilon J^{\beta} J^{\alpha} T(t)+O .
$$

In view of $(\mathrm{H} 2)$, we have

$$
\begin{gathered}
|x(t)-y(t)| \leq \epsilon J^{\beta} J^{\alpha} T(t)+N_{1}\|x-y\|_{X} \leq \epsilon \lambda_{T, \beta+\alpha} T(t)+N_{1}\|x-y\|_{X}, \\
\text { which implies that }|x(t)-y(t)| \leq \epsilon \lambda_{T, \beta+\alpha} T(t)+N_{1}\|x-y\|_{X} .
\end{gathered}
$$

On the other hand, by integrating and differentiating (6), we get

$$
M_{2} \leq \epsilon J^{\beta} T(t)
$$

Also, we can show that

$$
\left|D^{\alpha} x-D^{\alpha} y\right| \leq \epsilon \lambda_{T, \beta} T(t)+N_{2}\|x-y\|_{X} .
$$

We have also

$$
M_{3} \leq \epsilon J^{\alpha+\beta-\gamma} T(t) .
$$


By the same arguments as before, we observe that

$$
\left|D^{\gamma} x(t)-D^{\gamma} y(t)\right| \leq \epsilon \lambda_{T, \alpha+\beta-\gamma} T(t)+N_{3}\|x-y\|_{X} .
$$

Using the inequalities (11), (12) and (13) yields

$$
\begin{cases}|x(t)-y(t)| \leq \epsilon \max \left(\lambda_{T, \alpha+\beta}, \lambda_{T, \beta}, \lambda_{T, \alpha+\beta-\gamma}\right) T(t)+N_{1}\|x-y\|_{X}, & t \in I, \\ \left|D^{\alpha} x(t)-D^{\alpha} y(t)\right| \leq \epsilon \max \left(\lambda_{T, \alpha+\beta}, \lambda_{T, \beta}, \lambda_{T, \alpha+\beta-\gamma}\right) T(t)+N_{2}\|x-y\|_{X}, & t \in I, \\ \left|D^{\gamma} x(t)-D^{\gamma} y(t)\right| \leq \epsilon \max \left(\lambda_{T, \alpha+\beta}, \lambda_{T, \beta}, \lambda_{T, \alpha+\beta-\gamma}\right) T(t)+N_{3}\|x-y\|_{X}, & t \in I .\end{cases}
$$

Hence, it follows that there exists a real number

$$
\sigma=\frac{\max \left(\lambda_{T, \alpha+\beta}, \lambda_{T, \beta}, \lambda_{T, \alpha+\beta-\gamma}\right)}{1-N}
$$

such that

$$
\|x-y\|_{X} \leq \sigma \epsilon T(t), \quad t \in I
$$

Consequently, the problem (1) shows the Ulam-Hyers-Rassias stability.

\section{Numerical simulations}

In this section, we recall a numerical approach for the Caputo derivative. Then, for some fixed parameters, we investigate behavior of the above fractional Lane-Emden problem. To do this, we shall first obtain a reduced fractional differential system that is equivalent to our studied problem. Using a fourth-order Runge-Kutta integrator, the numerical simulations recover the convective behavior of the integer model in astrophysics [4]. In order to ensure the effect of the fractional order in Lane-Emden dynamics, we consider judicious values for $\alpha$ and $\beta$.

- Hydrodynamic simulations of giant stars, where the stellar profiles can be modeled in $[12,22,28]$ as

$$
\frac{1}{t} \frac{d}{d t}\left(t^{2} \frac{d y}{d t}+t^{2} \frac{g_{c}(a t)}{4 a \pi G p_{0}}\right)+y^{n}=0
$$

where $y$ is the polytropic temperature with index $n, t \equiv \frac{r}{a}$, and $p_{0}$ the central gas density. For $r \leq \frac{h}{2}$ and $x \equiv \frac{r}{h}$, the smoothed gravitational force of the core is defined by

$$
g_{c}(r):=\operatorname{Gm}_{c} \frac{x\left(\frac{32}{3}+x^{2}\left(\frac{-192}{5}+32 x\right)\right)}{h^{2}} .
$$

- Self-similar profiles of nonlinear wave equations in flat space-time were modeled in $[4,17]$ as

$$
\left(1-t^{2}\right) \frac{d^{2} y}{d t^{2}}+\left(\frac{A}{t}+B t\right) \frac{d y}{d t}-C y+D y^{E}=0
$$




\subsection{Numerical approach for Caputo derivative}

In this subsection, we presented an important numerical approach for the RiemannLiouville fractional integral and the Caputo derivative; we recall the theorems of $[6,19]$.

Theorem 9 Assume that $y \in \mathcal{C}^{1}([0,1], \mathbb{R})$. The fractional integration approach is given by

$$
J^{\alpha} y\left(t_{i}\right) \simeq \frac{h^{\alpha}}{\Gamma(\alpha+2)} \sum_{j=0}^{i} y\left(t_{j}\right) \sigma_{j}(\alpha), \quad i=0, \ldots, n+1,
$$

where

$$
\sigma_{j}(\alpha)= \begin{cases}(n+2-j)^{(\alpha+1)}+(n-j)^{(\alpha+1)}-2(n-j+1)^{(\alpha+1)}, & j=1, \ldots, i-1, \\ (n)^{(\alpha+1)}-(n-\alpha)(n+1)^{\alpha}, & j=0, \text { and } 1, j=i .\end{cases}
$$

Theorem 10 Assume that $y \in \mathcal{C}^{1}([0,1], \mathbb{R})$ and $0<\alpha \leq 1$. Then we have

$$
D^{\alpha} y\left(t_{i}\right) \simeq \frac{h^{1-\alpha}}{\Gamma(1-\alpha+2)} \sum_{j=0}^{i} y^{(j)}\left(t_{j}\right) \sigma_{j}(1-\alpha), \quad i=0, \ldots, n,
$$

where

$$
y^{(j)}=\left\{\frac{y_{1}-y_{0}}{h}, \quad j=0, \quad \frac{y_{j+1}-y_{j-1}}{2 h}, \quad j=1, \ldots, i-1, \quad \frac{y_{i}-y_{i-1}}{h}, \quad j=i .\right.
$$

\subsection{Simulation for Lane-Emden behaviors}

We note that the problem (1) can be reduced to the following system:

$$
\begin{aligned}
D^{\beta} y(t)= & z(t), \\
D^{\alpha} z(t)= & -\frac{k}{t^{\lambda}} D^{\alpha} y(t)-a_{1} F\left(t, y(t), D^{\gamma} y(t), J^{p} y(t)\right) \\
& -a_{2} G\left(t, y(t), D^{\gamma} y(t)\right)-a_{3} H(t, y(t))+L(t) .
\end{aligned}
$$

In order to achieve the mentioned phenomena, we take $1<\alpha+\beta \leq 2$, and $\lambda=1$. Taking into account our problem parameters, three cases can be observed:

Case 1: $\alpha=\beta=1$, we get

$$
\begin{aligned}
D y(t)= & z(t), \\
D z(t)= & -\frac{k}{t^{\lambda}} D y(t)-a_{1} F\left(t, y(t), D^{\gamma} y(t), J^{p} y(t)\right) \\
& -a_{2} G\left(t, y(t), D^{\gamma} y(t)\right)-a_{3} H(t, y(t))+L(t) .
\end{aligned}
$$

Case 2: $0<\alpha \leq 1, \beta=1$, we obtain

$$
\begin{aligned}
& D y(t)=z(t), \\
& D^{\alpha} z(t)=-\frac{k}{t^{\lambda}} D^{\alpha} y(t)-a_{1} F\left(t, y(t), D^{\gamma} y(t), J^{p} y(t)\right) \\
& -a_{2} G\left(t, y(t), D^{\gamma} y(t)\right)-a_{3} H(t, y(t))+L(t) .
\end{aligned}
$$


As a consequence,

$$
\begin{aligned}
D y(t)= & z(t), \\
D z(t)= & D^{1-\alpha}\left(-\frac{k}{t^{\lambda}} D^{\alpha} y(t)-a_{1} F\left(t, y(t), D^{\gamma} y(t), J^{p} y(t)\right)\right. \\
& \left.-a_{2} G\left(t, y(t), D^{\gamma} y(t)\right)-a_{3} H(t, y(t))+L(t)\right) .
\end{aligned}
$$

Case 3: $0<\alpha \leq 1,1 \leq \beta \leq 2$, we have

$$
\begin{aligned}
D^{\beta} y(t)= & z(t), \\
D^{\alpha} z(t)= & -\frac{k}{t^{\lambda}} D^{\alpha} y(t)-a_{1} F\left(t, y(t), D^{\gamma} y(t), J^{p} y(t)\right) \\
& -a_{2} G\left(t, y(t), D^{\gamma} y(t)\right)-a_{3} H(t, y(t))+L(t),
\end{aligned}
$$

that is,

$$
\begin{aligned}
J^{2-\beta} D[D y(t)] & =z(t) \\
D^{\alpha} z(t)= & -\frac{k}{t^{\lambda}} D^{\alpha} y(t)-a_{1} F\left(t, y(t), D^{\gamma} y(t), J^{p} y(t)\right) \\
& \quad-a_{2} G\left(t, y(t), D^{\gamma} y(t)\right)-a_{3} H(t, y(t))+L(t) .
\end{aligned}
$$

Therefore,

$$
\begin{aligned}
& D y(t)=z(t), \\
& J^{2-\beta} D z=w(t), \\
& D^{\alpha} w(t)=-\frac{k}{t^{\lambda}} D^{\alpha} y(t)-a_{1} F\left(t, y(t), D^{\gamma} y(t), J^{p} y(t)\right) \\
& -a_{2} G\left(t, y(t), D^{\gamma} y(t)\right)-a_{3} H(t, y(t))+L(t) .
\end{aligned}
$$

Consequently,

$$
\begin{aligned}
& D y(t)= z(t), \\
& \begin{aligned}
D z(t)= & D^{2-\beta} w(t) \\
D w(t)= & D^{1-\alpha}\left(-\frac{k}{t^{\lambda}} D^{\alpha} y(t)-a_{1} F\left(t, y(t), D^{\gamma} y(t), J^{p} y(t)\right)\right. \\
& \left.\quad-a_{2} G\left(t, y(t), D^{\gamma} y(t)\right)-a_{3} H(t, y(t))+L(t)\right) .
\end{aligned}
\end{aligned}
$$

I: As a first simulation, we consider the hydrodynamic simulations of giant stars, where $k=2, p=\gamma=0.01$, and $f, H, G, H, L$ are given by

$$
a_{1} F\left(t, y(t), D^{\gamma} y(t), J^{p} y(t)\right)=\frac{16 a^{4} m_{c}}{\pi p_{0} h^{6}} t^{4}+\frac{289}{51 t}\left(J^{p} y(t)\right)^{n}
$$


Figure 1 Numerical simulation of Case 1 for different values of the polytropic index $n$ and $\alpha=\beta=1$

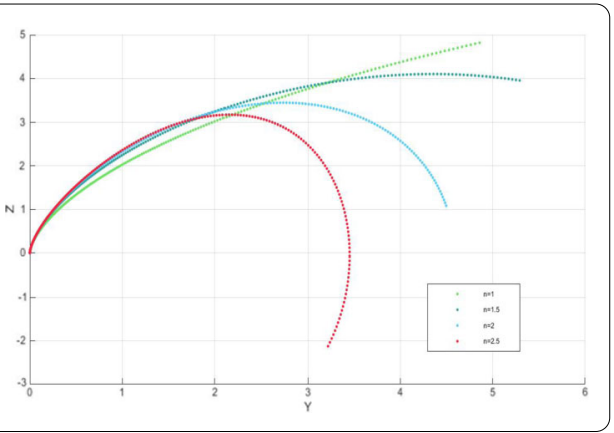

Figure 2 Numerical simulations of Case 2 for $\alpha=\{0.55,0.35,0.2\}$ and $\beta=1$

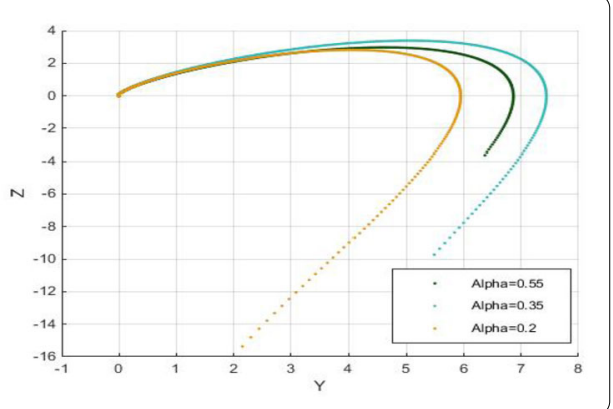

$$
\begin{aligned}
& a_{2} G\left(t, y(t), D^{\gamma} y(t)\right)=-\frac{48 a^{3} m_{c}}{\pi p_{0} h^{5}} t^{3}-\frac{663}{255 t}\left(D^{\gamma} y(t)\right)^{n}, \\
& a_{3} H(t, y(t))=\frac{32 a^{4} m_{c}}{\pi p_{0} h^{6}} t^{4}-\frac{527}{255 t}(y(t))^{n}, \\
& L(t)=\frac{8 a m_{c}}{\pi p_{0} h^{3}} t .
\end{aligned}
$$

For the first case, with initial conditions $(0,0), h=0.001$, and $n=\{1,1.5,2,2.5\}$, the numerical simulations are carried out only by the fourth-order Runge-Kutta method, for specific parameters, we have Fig. 1.

Remark 11 Through ongoing evaluation, we observe that the change in value of $n$ has no impact on the attitude of the remaining cases.

For the following simulation we take $n=1.5$ as it is more adequate. Now, to ensure that all three cases are convenient, we should be looking for a suitable fractional order.

For the second case, with initial conditions $(0,0), h=0.001$, and $\alpha=\{0.55,0.35,0.2\}$, numerical simulations are realized by a combination of the Caputo approach and the fourthorder Runge-Kutta method, we acquire Fig. 2. By comparing the above result with the one of the first case, we conclude that both cases are adequate for $\alpha=0.35$ (see Fig. 3).

For the third case, with initial conditions $(0,0,0.5), h=0.001, \beta=\{1.45,1.3,1.05\}$, for any $\beta$ value, we take $\alpha=\{0.55,0.35,0.2\}$. Numerical simulations are carried out by a combination of the Caputo approach and the fourth-order Runge-Kutta method, we see, according to Figs. $4-6$, that $\beta=1.3$ is the valid value. It is obvious from Fig. 7 that $\alpha=0.35$ is the appropriate value. 
Figure 3 Comparative simulation (Case 1-Case 2)

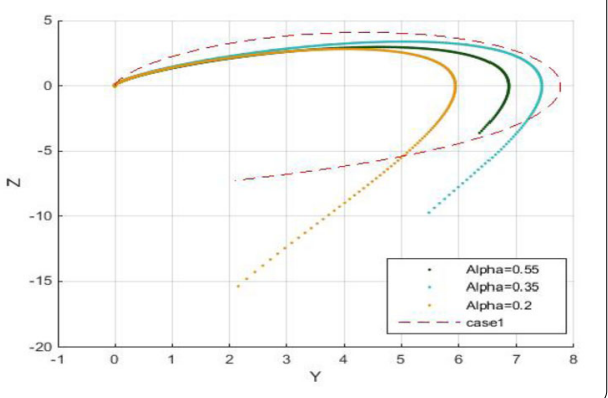

Figure 4 Numerical simulations of Case 3 for $\beta=1.45$ and $\alpha=\{0.55,0.35,0.2\}$

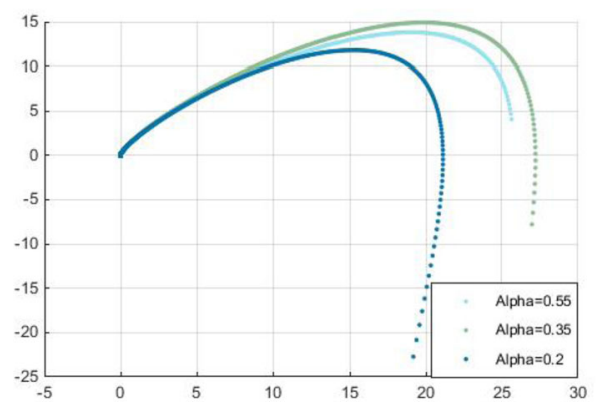

Figure 5 Numerical simulations of Case 3 for $\beta=1.3$ and $\alpha=\{0.55,0.35,0.2\}$

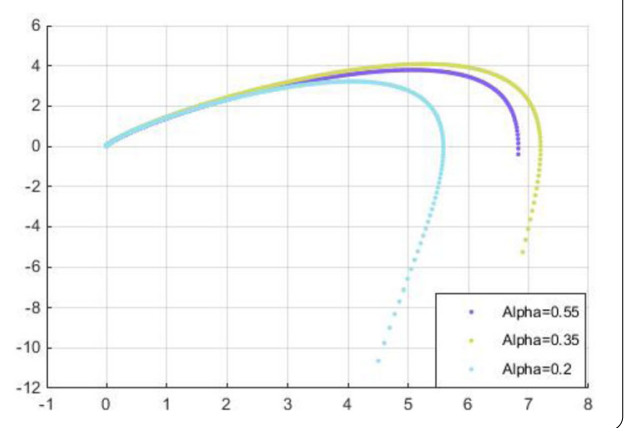

Figure 6 Numerical simulations of Case 3 for $\beta=1.05$ and $\alpha=\{0.55,0.35,0.2\}$

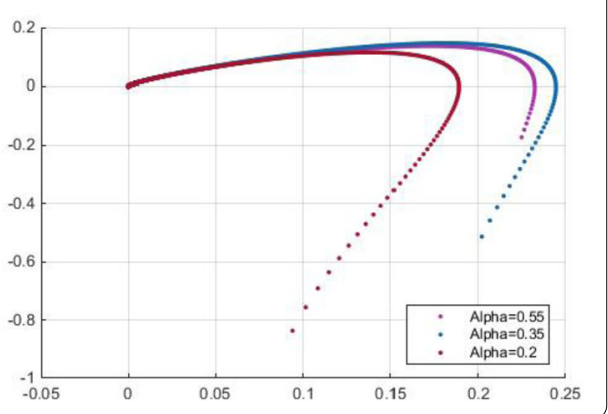

II: As a second simulation, we consider self-similar profiles of nonlinear wave equation in flat space-time, where $k=A, p=\gamma=0.01$, and $f, H, G, H, L$ are given by

$$
a_{1} F\left(t, y(t), D^{\gamma} y(t), J^{p} y(t)\right)=\frac{C}{1-t^{2}} J^{p} y(t)
$$


Figure 7 Comparative simulation (Case 1-Case 3)

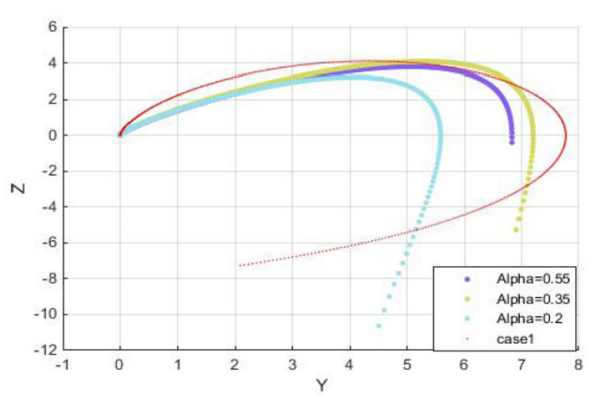

Figure 8 Numerical simulation of Case 1

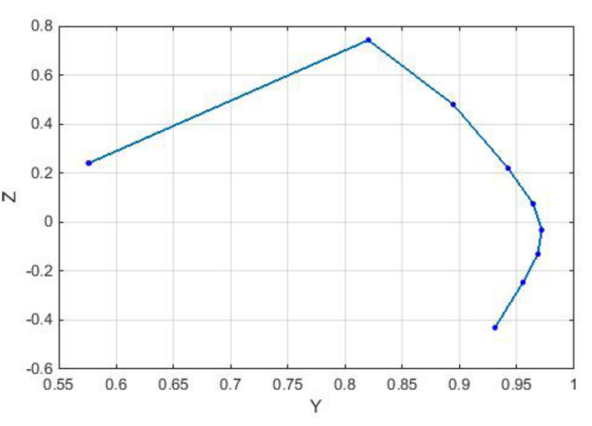

Figure 9 Numerical simulation of Case 2

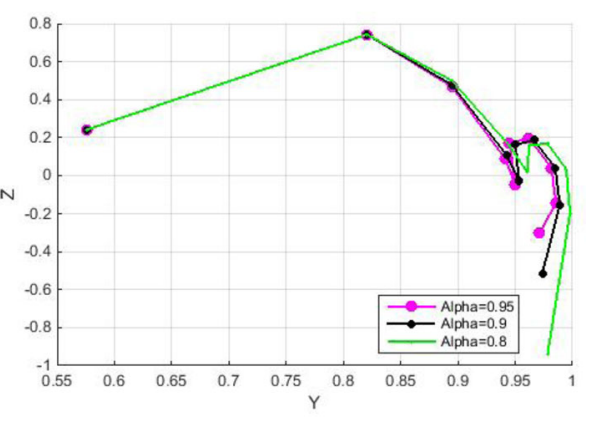

$$
\begin{aligned}
& a_{2} G\left(t, y(t), D^{\gamma} y(t)\right)=-\frac{B t}{1-t^{2}} D^{\gamma} y(t), \\
& a_{3} H(t, y(t))=-\frac{D}{1-t^{2}}(y(t))^{E}, \\
& L(t)=0
\end{aligned}
$$

with initial conditions $(0.576037116,0.24090)$, and $A=2, B=\frac{-25}{12}, C=\frac{1}{4}, D=1, E=2$, $h=0.01$. The integration for the first case is carried out by the fourth-order Runge-Kutta method, now, we are trying to determine an appropriate fractional order (see Fig. 8).

For the second case, we take the same data as above, and $\alpha=\{0.95,0.9,0.8\}$. Numerical simulations are realized by a combination of the Caputo approach and the fourth-order Runge-Kutta method (see Fig. 9).

Comparing our outcome to that in the first case, we summarize that the two cases are consistent in terms of $\alpha=0.95$ (see Fig. 10). 
Figure 10 Comparative simulation (Case 1-Case 2)
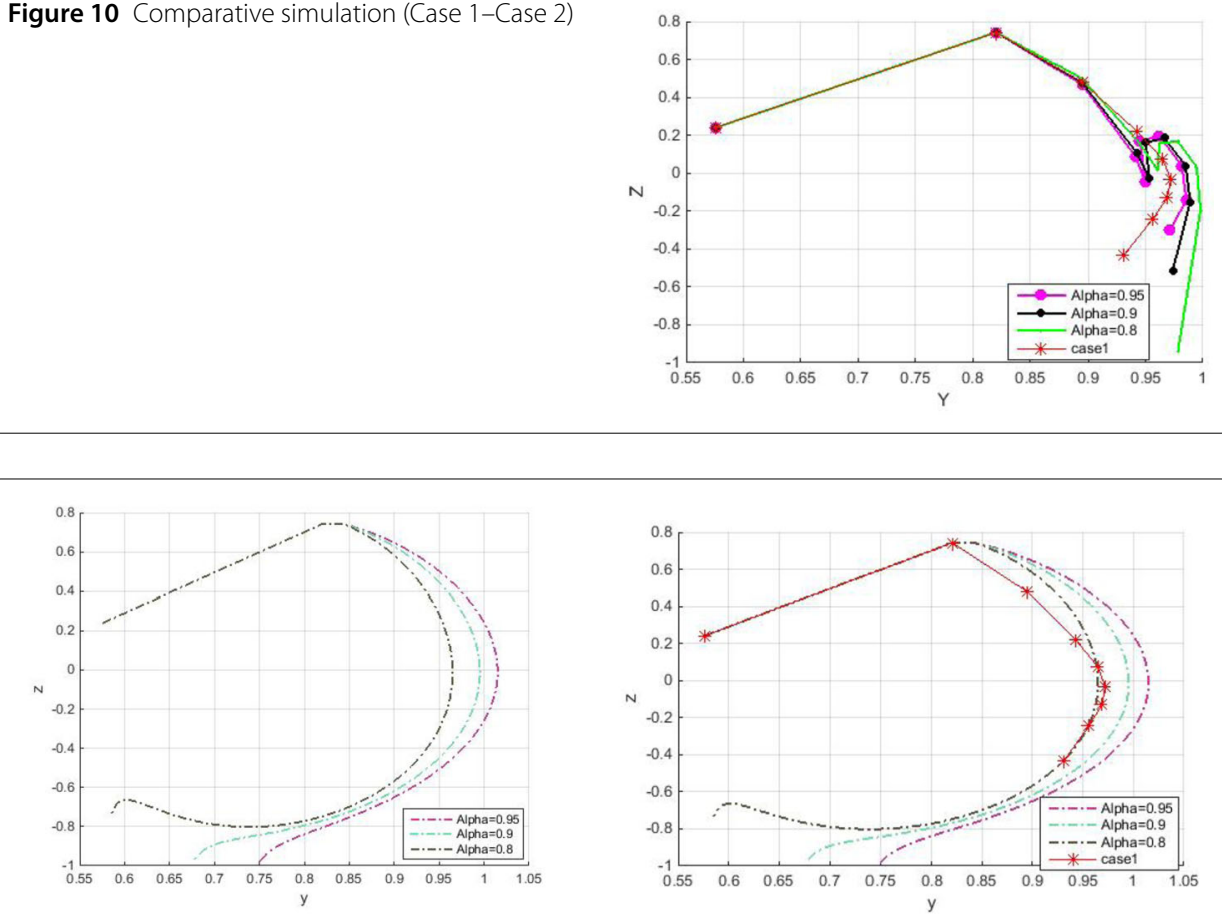

Figure 11 Numerical simulations of Case 3 for $\beta=1.2$ and different values of $\alpha$, on the left side. Comparative simulation (Case 1-Case 3), on the right side
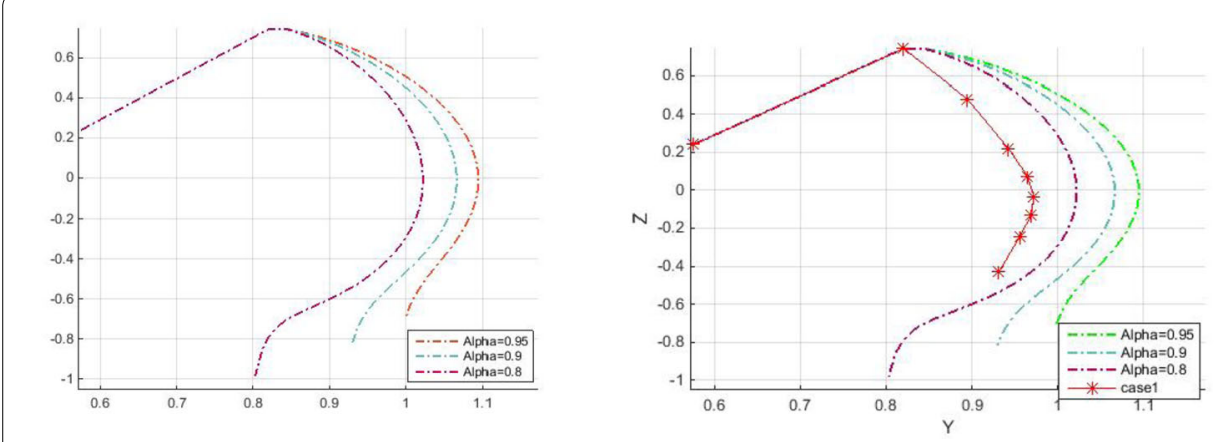

Figure 12 Numerical simulations of Case 3 for $\beta=1.15$ and different values of $\alpha$, on the left side. Comparative simulation (Case 1-Case 3), on the right side
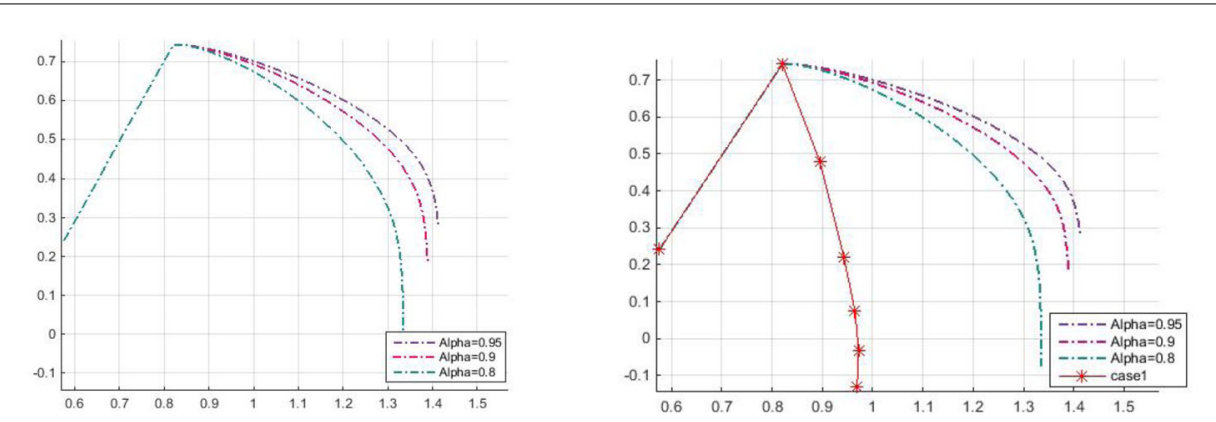

Figure 13 Numerical simulations of Case 3 for $\beta=1.05$ and different values of $\alpha$, on the left side. Comparative simulation (Case 1-Case 3), on the right side 
For the third case, with initial conditions $(0.576037116,0.24090,0)$, and $h=0.01, \beta=$ $\{1.2,1.15,1.05\}$, for each $\beta$, we take $\alpha=\{0.95,0.9,0.8\}$. Numerical simulations are carried out by a combination of the Caputo approach and the fourth-order Runge-Kutta method. It appeared from Figs. $11-13$ that $\beta=1.2$ and $\alpha=0.8$ are the most acceptable values too.

\section{Conclusions}

In this manuscript, we study some types of Ulam stability for a nonlinear fractional differential equation of Lane-Emden type with antiperiodic conditions. Then, by using a numerical approach for the Caputo derivative, we investigate the behaviors of the considered system.

\section{Acknowledgements}

The authors would like to express their sincere thanks to the editor and the anonymous reviewers for their helpful comments and suggestions.

\section{Funding}

There is no funding for this work.

Availability of data and materials

Not applicable.

\section{Competing interests}

The authors declare that they have no competing interests.

Authors' contributions

All authors contributed equally to the writing of this paper. All authors read and approved the final manuscript.

\section{Author details}

'Laboratory of Pure and Applied Mathematics, Faculty of Exact Sciences and Informatics, University of Mostaganem, Mostaganem, Algeria. ${ }^{2}$ Department of Mathematics, Faculty of Science and Arts, Düzce University, Düzce, Turkey.

\section{Publisher's Note}

Springer Nature remains neutral with regard to jurisdictional claims in published maps and institutional affiliations.

Received: 29 March 2021 Accepted: 23 June 2021 Published online: 09 July 2021

\section{References}

1. Agarwal, R.P., O'Regan, D.: Singular boundary value problems for superlinear second order ordinary and delay differential equations. J. Differ. Equ. 130, 333-355 (1996)

2. Agarwal, R.P., O'Regan, D., Staněk, S.: Positive solutions for mixed problems of singular fractional differential equations. Math. Nachr. 285(1), 27-41 (2012)

3. Bai, Z., Sun, W.: Existence and multiplicity of positive solutions for singular fractional BVPs. Comput. Math. Appl. 63(9), 1369-1381 (2012)

4. Bizon, P., Maison, D., Wasserman, A.: Self-similar solutions of semilinear wave equations with a focusing nonlinearity. Nonlinearity 20, 2061-2074 (2007)

5. Chandrasekhar, S.: An Introduction to the Study of Stellar Structure. Dover, New York (1967)

6. Dahmani, Z., Belhamiti, M.M., Sarikaya, M.Z.: A three fractional order Jerk equation with anti periodic conditions. Under review

7. Dahmani, Z., Taeb, A., Bedjaoui, N.: Solvability and stability for nonlinear fractional integro-differential systems of high fractional orders. Facta Univ., Ser. Math. Inform. 31(3), 629-644 (2016)

8. Emden, R.: Gaskugeln. Teubner, Leipzig (1907)

9. Ferraoun, S., Dahmani, Z:: Existence and stability of solutions of a class of hybrid fractional differential equations involving RL-operator. J. Interdiscip. Math. 23(4), 885-903 (2020)

10. Gouari, Y., Dahmani, Z., Sarikaya, M.Z.: A non local multi-point singular fractional integro-differential problem of Lane-Emden type. Math. Methods Appl. Sci. 43(11), 6938-6949 (2020)

11. Govindan, V., Hammachukiattikul, P., Rajchakit, G., Gunasekaran, N., Vadive, R.: A new approach to Hyers-Ulam stability of variable quadratic functional equations. J. Funct. Spaces 2021, 6628733 (2021)

12. Guidarelli, G., Nordhaus, J., Chamandy, L., Chen, Z., Blackman, E.G., Frank, A., Carroll-Nellenback, J., Liu, B.: Hydrodynamic simulations of disrupted planetary accretion discsinside the core of an AGB star. Mon. Not. R. Astron. Soc. 490, 1179-1185 (2019)

13. Hammachukiattikul, P., Unyong, B., Suresh, R., Rajchakit, G., Vadivel, R., Gunasekaran, N., Lim, C.P.: Runge-Kutta Fehlberg method for solving linear and nonlinear fuzzy Fredholm integro-differential equations. Appl. Math. Inf. Sci. 15(1) 43-51 (2021)

14. Hyers, D.H.: On the stability of the linear functional equation. Proc. Natl. Acad. Sci. USA 27, 222-224 (1941) 
15. Ibrahim, R.W.: Stability of a fractional differential equation. Int. J. Math. Comput. Phys. Quantum Eng. 7(3), 300-305 (2013)

16. Kilbas, A.A., Srivastava, H.M., Trujillo, J.J.: Theory and Applications of Fractional Differential Equations. Elsevier, Amsterdam (2006)

17. Kycia, R.A.: Perturbed Lane-Emden equations as a boundary value problem with singular endpoints. J. Dyn. Control Syst. 26, 333-347 (2020)

18. Lane, J.H.: On the theoretical temperature of the sun under the hypothesis of a gaseous mass maintaining its volume by its internal heat and depending on the laws of gases known to terrestrial experiment. Am. J. Sci. Arts s2-50, 57-74 (1870)

19. Li, C., Chen, A., Ye, J.: Numerical approaches to fractional calculus and fractional ordinary differential equation J. Comput. Phys. 230, 3352-3368 (2011)

20. Mechee, S.M., Senu, N.: Numerical study of fractional differential equations of Lane-Emden type by method of collocation. Appl. Math. 3, 851-856 (2012)

21. Mohanapriya, A., Ganesh, A., Rajchakit, G., Pinelas, S., Govindan, V., Unyong, B., Gunasekaran, N.: New generalization of Hermite-Hadamard type of inequalities for convex functions using Fourier integral transform. Thai J. Math. 18(3), 1051-1061 (2020)

22. Ohlmann, S.T., Röpke, F.K., Pakmor, R., Springel, V.: Constructing stable 3D hydrodynamical models of giant stars. Astron. Astrophys. 599, A5 (2017)

23. Okunuga, S.A., Ehigie, J.O., Sofoluwe, A.B.: Treatment of Lane-Emden type equations via second derivative backward differentiation formula using boundary value technique. In: Proceedings of the World Congress on Engineering IWCE, London, UK, pp. 4-6 (2012)

24. Rassias, T.M.: On the stability of the linear mapping in Banach spaces. Proc. Am. Math. Soc. 72, 297-300 (1978)

25. Tablennehas, K., Abdenebi, A., Dahmani, Z., Belhamiti, M.M.: An anti-periodic singular fractional differential problem of Lane-Emden type. J. Interdiscip. Math. (2021). https://doi.org/10.1080/09720502.2020.1848318

26. Ulam, S.M.: Problems in Modern Mathematics. Wiley, New York (1940)

27. Unyong, B., Govindan, V., Bowmiya, S., Rajchakit, G., Gunasekaran, N., Vadivel, R., Lim, C.P., Agarwal, P.: Generalized linear differential equation using Hyers-Ulam stability approach. AIMS Math. 6(2), 1607-1623 (2021)

28. Winkler, D.: Polytropes: Applications in Astrophysics and Related Fields, Chemistry in Australia (2005)

\section{Submit your manuscript to a SpringerOpen ${ }^{\circ}$ journal and benefit from:}

- Convenient online submission

- Rigorous peer review

- Open access: articles freely available online

- High visibility within the field

- Retaining the copyright to your article

Submit your next manuscript at $\gg$ springeropen.com 